\title{
MANAGING WORKFORCE RESOURCE ACTIONS WITH MULTIPLE FEEDBACK CONTROL SCHEMES
}

\author{
Young M. Lee \\ Lianjun An \\ Daniel Connors \\ IBM T.J. Watson Research Center \\ 1101 Kitchawan Road \\ Yorktown Heights, New York 10598, USA
}

\begin{abstract}
Demand disturbances in service businesses are typically managed by resource actions such as hiring, releasing and cross training of the workforce. The magnitudes of resource actions are often decided by estimating the discrepancy between the demand for services and the supply of workforce. However, naïve feedback control of the resource actions by policies that equate the discrepancy to the control action can produce undesirable effects such as oscillation between hiring and releasing of workforce, and amplified oscillation through the stages of the service processes. Effective combination of multiple feedback control schemes can produce desirable policies of workforce resource actions. In this work, we study application of control theoretic principles in managing resource actions to see how various feedback control schemes can improve costs, utilization and stability of workforce.
\end{abstract}

\section{INTRODUCTION}

Service businesses, such as business consulting, call centers, technical services and IT outsourcing, are growing rapidly (Dietrich et al. 2006), and have become a significant portion of the U.S. and world economy. Demand disturbance in service businesses occur as in other businesses, and it can produce undesirable effect for the businesses such as reduced service level, reduced utilization, oscillation of workforce availability and amplification. The phenomena have been studied widely in manufacturing supply chain, and it has been observed that the business impacts arising from these include excessive levels of inventory, poor customer services due to shortage or long backlog, and high costs for corrections (i.e., expedited shipment and over-time of workforce, etc.). Amplification and volatility of inventory as it moves through the supply chain is known as bullwhip effect and its impacts and remedies have been studied by many researchers including Forrester (1960), Sterman (1989), and Lee at al. (1997). However, the service supply chain is different from manufacturing supply chain due to the fact that the main resource is workforce not materials; therefore, the demand disturbances can manifest differently.

Services supply chain depends heavily on workforce, and unlike manufacturing supply chains, unused resource (workforce) cannot be carried over (thus perishable). Resource actions, such as hiring, releasing and cross training, take time (lead time) and incur monetary costs, for example employment agency search fees in the case of hiring or severance payments in the case of releasing resources. Availability of resources also degrades over time through attrition and termination of workforce, etc. Another unique attribute of workforce is that the skill levels and types change through service engagement experience and training. Therefore, the resource actions can be more complicated than material acquisition for inventory control in manufacturing supply chain.

When various disturbances (e.g., surge, step, ramp, oscillatory) in demand occur in service businesses, the disturbance is typically managed by resource actions such as hiring, releasing and cross training the workforce. The magnitudes of resource actions are often decided by estimating the discrepancy between the demand of services and the supply of workforce. However, naïve feedback control (hiring, releasing or cross training workforce by policies that are directly proportional to the discrepancy alone) can produce undesirable effects such as oscillation between hiring and releasing, and amplified oscillation through the stages of the service processes. Effective combination of multiple feedback control schemes can produce desirable policies of workforce resource actions.

Application of control theoretic principle to manage adverse effects has been studied in manufacturing supply chain (Simon 1952, Wikner 1991, White 1999 and Dejonckheere 2004 etc.). Ortega and Lin (2004) provides an excellent review of control theory applied to the production and inventory problem. However, little study has been done in services supply chain. Anderson \& Morrice $(1999,2000)$ modeled a simplified services supply chain for mortgage service processing with multiple stages of 


\section{Lee, An , and Connors}

application processing, credit checking, surveying and title checking, and observed oscillation and amplification through the stages. They also study the impact of resource hiring delay and engagement execution delay, and also noticed that information sharing can reduce the amplitude of oscillation. Akkermans et al. (2003) also observed amplification effects in a service supply chain for a telecommunication process, and identified that the root causes for the amplification effect are interdependence among workload, work quality and rework. They also observed that quality improvement throughout all stages of the supply chain can be an effective counter measure for the amplified oscillation of service backlog. However, no prior study has been done in computing multiple feedback control schemes by using combinations of proportional (P), derivative (D), and integral (I) control to determine effective policies of workforce resource actions.

The purpose of this work is to model and study how disturbances of demand (e.g., surge, step, ramp, oscillatory) impact services supply chain with respect to costs, utilization and stability of workforce, and to identify what control policies can mitigate the adverse effect by applying control theoretical principles. We attempt to characterize various control policies, PID controls, for workforce actions, i.e., the policies for hiring, releasing and cross training. We also attempt to determine conditions (and timing) under which specific control actions (resource policies) are beneficial, identify conditions for stability, and explore trade-off between responsiveness to demand and volatility of resource actions. Ultimately, this research work would identify good and bad polices (strategies) of resource actions of workforce for various demand disturbance situations, and generates useful insights for workforce management decision makers. It should be stressed that controller we described here is never meant to make decisions by itself on resource actions by replacing human decision makers. The control scheme can, however, provide useful information to the workforce resource managers as a decision support tool, so that good decisions on resource actions are made. This paper is focused on initial finding for one type of demand disturbance, i.e., step increase in demand.

\section{DESCRIPTION OF A SERVICE SUPPLY CHAIN MODEL}

\subsection{Single Stage Service Business Model}

We model a simplified services business using the system dynamics (SD) method (Sterman 2000). The model is developed using a system dynamics modeling tool called Vensim (Ventana Systems Inc. 1998) as shown in Fig.1. The business we modeled here is one with service engagements, which require a certain number of workforce for a certain duration such as IT service and business con- sulting, etc. Figure 1 is a simplified view of the SD model without showing many parameters.

There are two streams of activities in the model. The first one is the flow of service engagements coming into a service firm as shown in the top portion of Figure 1. The backlog of service engagements accumulates based the difference between inflow of demand arrival, $A r_{t}$, and execution rate, $E r_{t}$. In this model, we assume that all the service engagement is ultimately executed although some engagements would be delayed due to capacity shortages as shown in the expression of, $E r_{t}$, in (1).

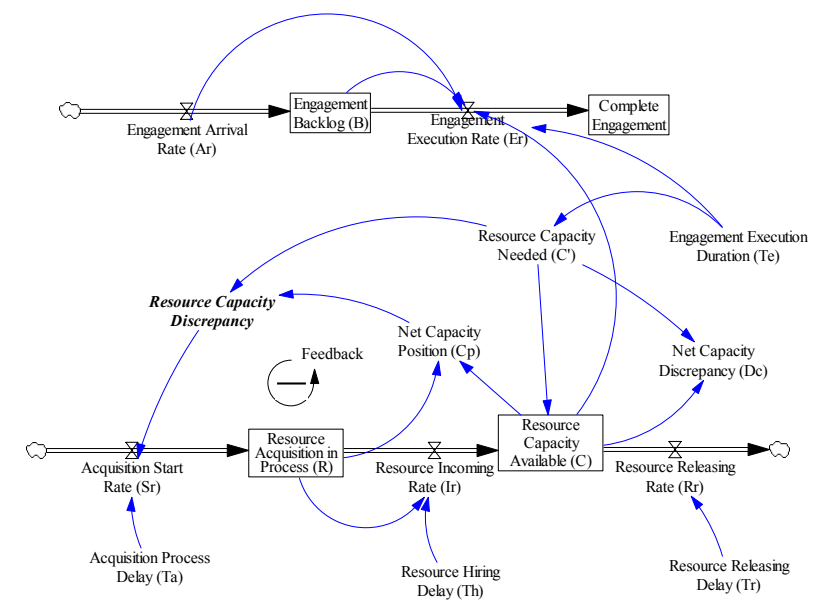

Figure 1: A Single Stage Service Business Model

$$
B_{t}=\int_{0}^{t}\left(A r_{\tau}-E r_{\tau}\right) d \tau+B_{0}
$$

where $B_{t}=$ demand backlog at $t$

$A r_{t}=$ arrival rate of demand at $t$

$E r_{t}=$ execution rate of demand at $\mathrm{t}$

$=\min \left(C_{t}, B_{t} / T e+A r_{t}\right)$

$C_{t}=$ resource capacity available at $t$

$T_{e}=$ delay of fulfilling demand engagement

$B_{0}=$ initial backlog at $t=0$

The second flow in the model is the flow of workforce as shown in the bottom part of the model in Figure 1. The resource capacity available, $C_{t}$, accumulates based on the difference between the resource incoming (employees joining the service firm) rate, $I r_{t}$, and the resource releasing rate, $R r_{t}$, as shown in (2).

$$
C_{t}=\int_{0}^{t}\left(I r_{\tau}-R r_{\tau}\right) d \tau+C_{0}
$$

Where

$C_{t}=$ resource capacity available at $t$ 
$I r_{t}=$ resource incoming rate at $t$

$=R_{t} / T_{h}$

$R_{t}=$ resource acquisition in-process at $t$

$T_{h}=$ delay in resource hiring

$R r_{t}=$ resource releasing rate at $t$ $=D c_{t}^{+} / T_{r}$

$D c_{t}^{+}=$net capacity discrepancy (positive part) at $t$

$T_{r}=$ delay in resource releasing

$C_{0}=$ initial resource capacity at $t=0$

The resource incoming rate, $I r_{t}$, in turn, is modeled as resource acquisition in process, $R_{t}$, (number of employees in the hiring process pipeline) divided by the delay in resource hiring, $T_{h}$, (time needed to bring in the workforce once the hiring process is in place). The resource releasing rate, $R r_{t}$, in turn, is modeled as net capacity discrepancy, $D c_{t}{ }^{+}$, divided by delay in resource releasing (time needed to release workforce), $T_{r}$. The net capacity discrepancy, $D c_{t}$, is the resource capacity available minus the resource capacity needed.

The resource acquisition in process, $R_{t}$, in turn, accumulates based on the difference between resource acquisition start rate, $S r_{t}$, and resource incoming rate, $I r_{t}$, as shown in (3).

$$
R_{t}=\int_{0}^{t}\left(S r_{\tau}-I r_{\tau}\right) d \tau+R_{0}
$$

Where $S r_{t}=$ resource acquisition start rate at $t$

$$
R_{0}=\text { initial resource acquisition start rate at } t=0
$$

The resource acquisition start rate, $S r_{t}$, is the key control variable (as explained later in the control system section of the paper) for deciding how many people to hire, and it depends on the discrepancy of resource capacity, $\varepsilon_{t}$, between supply and demand (as shown in (4)), and control parameters such as the gain constant, $K_{c}$, which is explained below.

$$
\varepsilon_{t}=C_{t}^{\prime}-C p_{t}
$$

$$
\text { Where } \begin{aligned}
C_{t}{ }^{\prime} & =\text { resource capacity needed at } t \\
& =B_{t} / T_{e} \\
C p_{t} & =\text { net capacity position at } t \\
& =R_{t}+C_{t}
\end{aligned}
$$

The gain constant, $K_{c}$, is typically treated to be 1 (as in the work of Anderson et al. (1999, 2000), and effect of various values of $K_{c}$ will be explained in the subsequent sections below. The overall decision of resource acquisition start rate, $S r_{t}$, is delayed (divided) by the delay for resource acquisition start rate $T_{a}$.

$$
\begin{gathered}
\qquad r_{t}=K_{c} \cdot \varepsilon_{t} / T_{a} \\
\text { Where } \varepsilon_{t}=\text { resource capacity discrepancy } \\
\text { between supply and demand } \\
K_{c}=\text { gain (sensitivity) constant } \\
T_{a}=\text { delay for resource acquisition process }
\end{gathered}
$$

\subsection{Multi-Stage Service Business Model}

The service supply chain we modeled here is for an IT software development service, which consists of three stages, each with a finite workforce capacity handing different tasks as shown in Figure 2. Its structure is similar to the model described by Anderson et al. (1999, 2000) and Akkerman et al. (2003). In the initial stage, pressales service opportunities are coming to a service firm and are accumulated as potential service engagements. The first phase that involves workforce is the contracting phase, which uses sales personnel and deals with designing the contract, pricing and scoping, etc. The second stage is the consulting stage, which uses business consultants to design tools, make project plans, etc. The third stage is the development stage, which uses engineers to develop and deploy the software. Each of the stages has its own details described in the single stage model in the previous section 2.1. Each stage is responsible for its own resource actions, i.e., hiring and releasing workforce. For simplicity, we assume that there are no dropped or lost opportunities and all of the service opportunities are all eventually engaged. For this paper, we also assume that resources are not shared between the stages, although we plan to extend our research to explore the impact of sharing of resources between stages of service process.

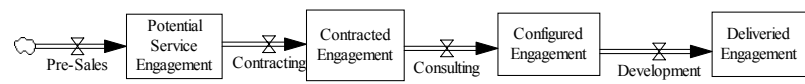

Figure 2: Multi-Stage Service Business Model

\section{SIMULATION ANALYSIS}

The system dynamics model described in the previous section is simulated for 356 days. The following parameters are used in the simulation:

- Engagement arrival rate $\left(A r_{t}\right): 20$ [\# of engagements/day]

- Delay of fulfilling each demand engagement $\left(T_{e}\right): 5$ [day]

- $\quad$ Resource Hiring Delay $\left(T_{h}\right): 30$ [days] 
- Resource Releasing Delay $\left(T_{r}\right): 14$ [days]

- Unit Hiring Costs: 10,000 [\$]

- Unit Releasing Cost: 20,000 [\$]

- Unit Service Penalty Cost: 1,000 [\$]

The demand disturbance modeled here is a step increase, i.e., at day 50 , the demand arrival rate increases from 20 [\# of engagements/day] to 25 . The demand profile is shown in Figure 3. The resource acquisition start rate, $S r_{t}$, is decided by the discrepancy of resource capacity between supply and demand with the gain constant (also called proportional control constant) value of $1\left(K_{c}=\right.$ 1). The setting is similar to the model described by Anserson et al. $(1999,2000)$.

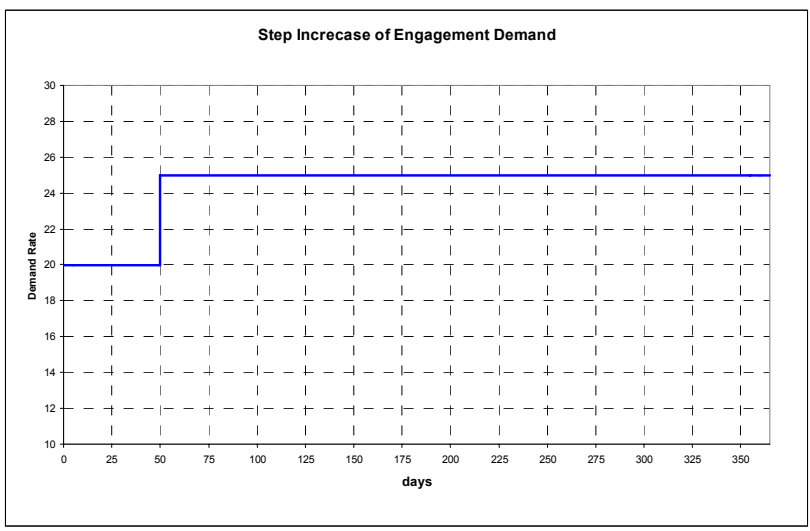

Figure 3: Step Increase of Engagement Demand

\subsection{Simulation of Single Stage Model}

In this section, we present simulation result for a single stage model. The general phenomenon is the same for all the stages. Figure 4 shows the simulation result of engagement backlog, which has a steady state initial value of 100 engagements, and then at day 50 it starts to oscillate. Figure 5 shows simulation result of net capacity discrepancy, which is the resource capacity needed minus the resource capacity available. The positive side of the curve indicates a shortage and the negative side represents a surplus of resource capacity. Figure 6 shows the resource incoming rate and the resource releasing rate. The resource incoming rate is the rate of workforce joining the firm as a result of hiring in process. Once acquisition starts $\left(S r_{t}\right)$, the resources become available according to exponential decay model. The hiring in-process (in pipeline) has the hiring lead time, $T_{h}$, and at the end of lead time, a part of workforce actually joins the firm as available resource. The resource releasing rate is the rate of workforce being released based on the negative side of the net capacity discrepancy (Figure 5). Note that the resource incoming rate and releasing rate overlap due to the lead time of hiring. When there is a surplus of workforce and newly hired workforce is in the pipeline, the releasing action will still have to be used to prevent an excessive workforce. Therefore, as new workforce joins the firm, releasing can occur at the same time. However, the resource acquisition start rate $\left(S r_{t}\right)$ would never overlap with the resource releasing rate because no hiring action should be initiated when workforce surplus is observed.

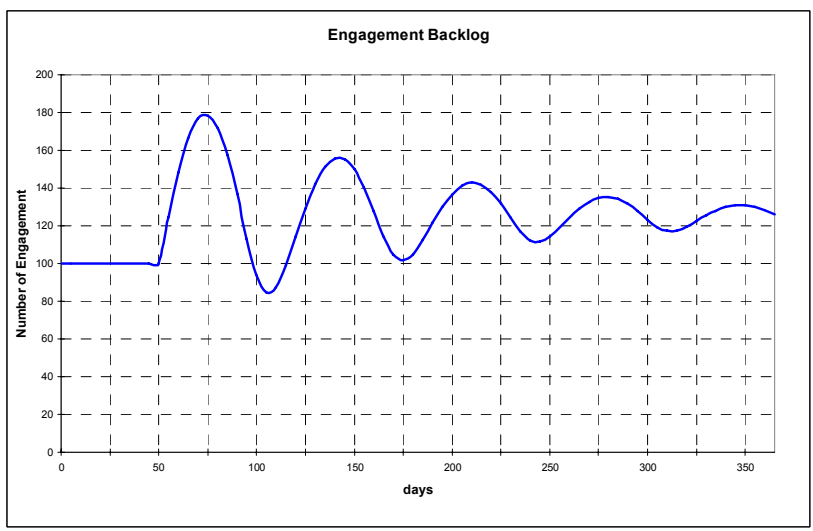

Figure 4: Engagement Backlog for Single Stage

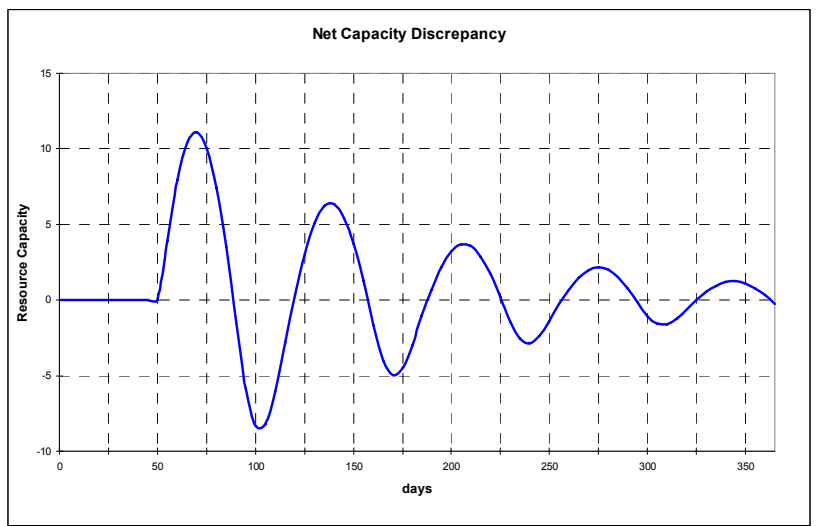

Figure 5: Net Capacity Discrepancy for Single Stage

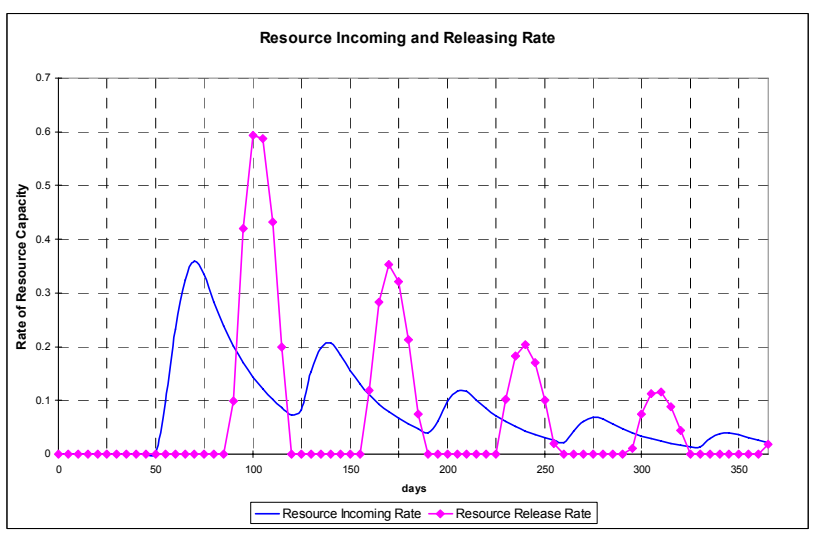

Figure 6: Resource Incoming Rate and Releasing Rate 
As can be seen in Figure 4, the engagement backlog starts to grow, as the demand step increase occurs at $\mathrm{T}=50$ days, and the net capacity discrepancy (shortage) also increases as shown in Figure 5. Due to the resource capacity action, $S r_{t}$, resources start to join the firm ant $\mathrm{T}=50$, and gradually increase and peak at $\mathrm{T}=70$ as shown in Figure 6. The available resource (through hiring) finally catches up with the needed resource, i.e., the net capacity discrepancy becomes zero at $\mathrm{T}=87$ days; however, the incoming rate remains positive in order to eliminate the backlog that still exists. At the around the same time ( $\mathrm{T}=87$ days), the releasing rate starts to kick in to eliminate the unnecessary resource.

The costs that are computed in the simulation model to compare different scenarios and effectiveness of control schemes consist of unit hiring cost of $\$ 10,000$, unit releasing cost of $\$ 20,000$ and unit service penalty cost of $\$ 1,000$. The service penalty cost occurs when an engagement is delayed due to the shortage of workforce. The accumulated costs for the scenario are $\$ 1,394,000$.

\subsection{Simulation of Multi-Stage Model}

The multi-stage simulation includes three stages of the service business; contracting stage, consulting stage and development stage. Figure 7 shows the engagement backlog changing over time for the three stages. The potential engagement backlog corresponds to the backlog in the single stage model in the previous section.

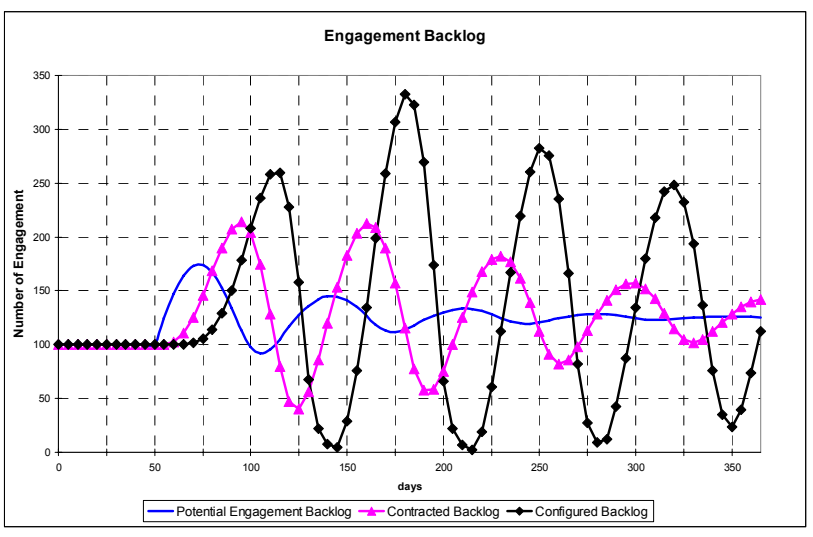

Figure 7: Engagement Backlog for Multi-Stage

The engagement backlog for the second stage, the contracted backlog, is higher than the first stage, and the backlog of the third stage (configured backlog) is, in turn, even higher than the second stage. The oscillation of the backlog is amplified as it moves through the stages of the service supply chain. This phenomenon is similar to the bullwhip effect observed in the supply chain (Forrester 1960, Sterman 1989, and Lee at al. 1997). This is also very similar to the amplification effect in a service supply chain model observed by Anderson et al. (1999, 2000). For the simplicity, the control study is limited to the single stage model in this paper.

\section{DESCRIPTION OF FEEDBACK CONTROL SYSTEM}

An overall picture of the relationships among variables in the services supply chain can be represented by a block diagram as shown in Figure 8. (See D'Azzo and Houpis (1981) for principles of control theory and systems.) The diagram shows the flow of information around the control system and the function of each part of the system. The needed capacity (set point) and the available capacity enter the comparator, and their difference, the error, leaves the comparator and enters the controller. The set point is the desired value of the systems variables, resource capacity needed, that need to be controlled. The error is the discrepancy in resource capacity shown in (4).

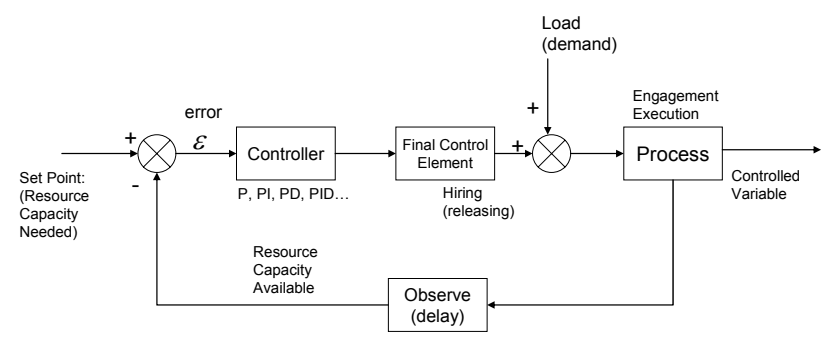

Figure 8: Block Diagram for the Control System

The controller uses the error and computes control actions that are proportional (P), derivative (D), or integral (I) or any other combination such as PI (proportionalintegral) and PID (proportional-integral-derivative). Using the control action computed by the controller, the final control element determines the resource actions of hiring or releasing. The new demand (load) and the resource action go into the process, and the available resource capacity is observed, and is fed back to the controller as a feedback control (also called closed-loop system). The process here is the process defined in earlier section in Figure 1 and 2.

The control variable here is the resource acquisition start rate, $S r_{t}$, and it can be a combination of P, D, I controls as shown in (6). The proportional controller produces a resource action which is proportional to the error $\varepsilon$ (also called discrepancy) as shown in the first term in (6). The second term is the derivative control which is derivative of the error, and the third term is the integral control which is based on the integral of the error. The controller can be combinations of PI, PD or PID. 


$$
S r_{t}=\left(K_{c} \varepsilon+K_{c} \tau_{D} \frac{d \varepsilon}{d t}+\frac{K_{c}}{\tau_{I}} \int_{0}^{t} \varepsilon \cdot d t\right) / T_{a}
$$

$$
\begin{aligned}
& \text { Where } \varepsilon_{t}=\text { resource capacity discrepancy } \\
& \text { between supply and demand } \\
& K_{c}=\text { gain (sensitivity) constant } \\
& T_{d}=\text { derivative time constant } \\
& T_{i}=\text { integral time constant } \\
& T_{a}=\text { delay for resource acquisition process }
\end{aligned}
$$

For the service supply chain models (in both singlestage or multiple-stage) used in the simulation analysis in Section 3, the control variable (the resource acquisition start rate, $\left.S r_{t}\right)$ uses only proportional control with the gain constant (also called proportional control constant) value of $1\left(K_{c}=1\right)$. The service supply chain modeled by Anserson et al. $(1999,2000)$, therefore, used a special case $\left(K_{c}=1\right)$ of proportional control. In our work, we explore more effective control schemes (P, I, D controls) for the service supply chain.

\section{CONTROL SCHEMES \& ANALYSIS}

The control variable here is the resource acquisition start rate, $S r_{t}$ Therefore, the controller adjusts $S r_{t}$ to eliminate the error, which is in this case the resource capacity discrepancy. It seems natural to think that the controller should change the resource acquisition start rate by an amount proportional to the error as shown by Anderson et al. (1999, 2000); however, that is not always effective as we shall see below. Since the controller uses information about the deviation of the system from its desired state (zero error) to control the system, the system is a feedback control system.

\subsection{Effect of Proportional Control}

We used various degree of proportional control, i.e., started with $K_{c}=1$, then increased the $K_{c}$ gradually to 50 . Figure 9 shows the profiles of backlog for $K_{c}=1,10,30$ and 50, and Table 1 shows the total costs for the four $K_{c}$ values. At first glance, it seems that the larger the value of $K_{c}$, the better the control. From $K_{c}=1$ to $K_{c}=10$, the magnitude of the backlog oscillation becomes smaller, and total cost decreases to $\$ 835,000$ from $\$ 1,396,000$. However, as $K_{c}$ increase beyond 10, other problems appear. At $K_{c}=30$ and 50, as shown in Figure 9, the oscillation of backlog fluctuates more (in reverse shape, though), and the resulting total cost increases to $\$ 1,601,00$ for $K_{c}=30$ and $\$ 3,489,000$ for $K_{c}=50$ due to the oscillation of hiring and releasing of workforce and the penalty of delayed execution of engagements. High values of $K_{c}$ have the tendency of over-adjusting. That is, it is too sensitive to demand disturbance which would tend to disappear in time even with smaller $K_{c}$. This causes an undesirable effect of fluctuating the backlog. The fluctuations are called noise. Therefore, as $K_{c}$ is increased, the system becomes over-sensitive causing fluctuation of systems variables.

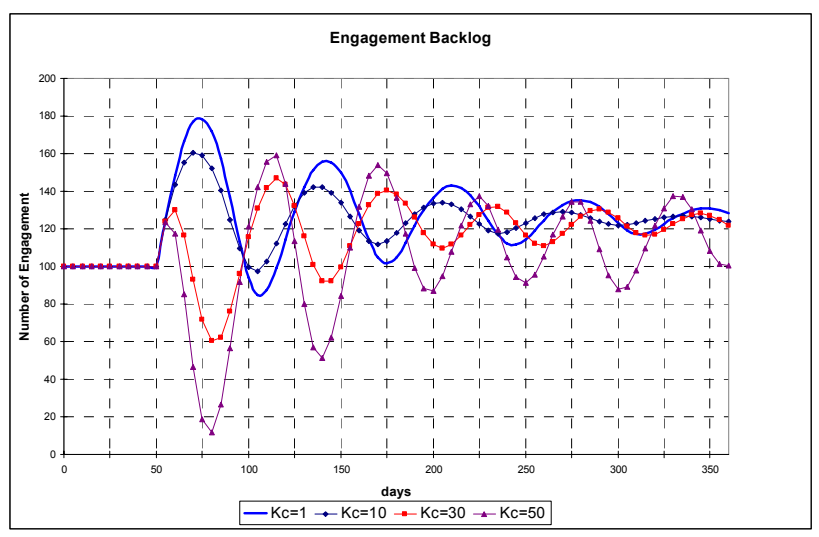

Figure 9: Effect of Proportional Control

Table 1: Total Costs for Various P-Control Parameters

\begin{tabular}{|l|r|r|r|}
\hline P-Control & D-Control & I-Control & \multicolumn{1}{c|}{ Costs } \\
\hline$K_{c}=1$ & None & None & $\$ 1,396,000$ \\
\hline$K_{c}=10$ & None & None & $\$ 835,000$ \\
\hline$K_{c}=30$ & None & None & $\$ 1,601,000$ \\
\hline$K_{c}=50$ & None & None & $\$ 3,489,000$ \\
\hline
\end{tabular}

\subsection{Effect of Derivative Control}

In this section, we add to the proportional control a derivative control term, which is proportional to the derivative of the error. Therefore, the control system is PD (Proportional-Derivative), and the control equation would include only first two terms in (6). We used a constant proportional control $\left(K_{c}=1\right)$, but increased the derivative term, $T_{d}$, from 0 to 5,10 and 30 .

Derivative control typically stabilizes the response of the system by reducing the rate of change of system variables. As it can be seen in Figure 10, as $T_{d}$ increases from 0 to 5 and 10 , the amplitude of the oscillations reduces, and the total costs reduce from $\$ 1,396,000$ to $\$ 833,000$ and to $\$ 593,000$ (see Table 2), which is much better than the minimum costs we obtained from the proportional control. However, as $T_{d}$ becomes very large (e.g., $T_{d}=30$ ), although the stability is improved, the total cost increases to $\$ 1,592,000$ as the control system now incurs large release costs. 


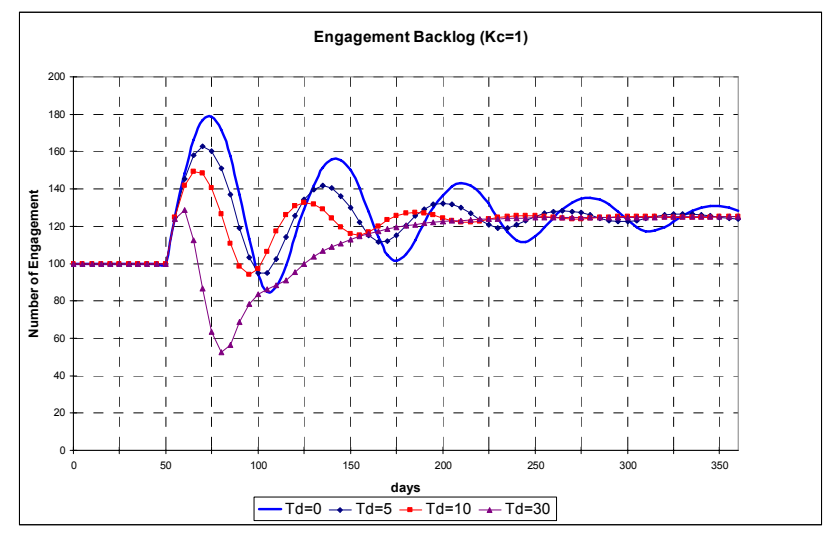

Figure 10: Effective of Derivative Control

Table 2: Total Costs for Various D-Control Parameters

\begin{tabular}{|l|l|r|r|}
\hline P-Control & D-Control & I-Control & \multicolumn{1}{|c|}{ Costs } \\
\hline$K_{c}=1$ & $T_{d}=0$ & None & $\$ 1,396,000$ \\
\hline$K_{c}=1$ & $T_{d}=5$ & None & $\$ 833,000$ \\
\hline$K_{c}=1$ & $T_{d}=10$ & None & $\$ 593,000$ \\
\hline$K_{c}=1$ & $T_{d}=30$ & None & $\$ 1,592,000$ \\
\hline
\end{tabular}

\subsection{Effect of Integral Control}

With proportional action only, the control system is able to change the control variable and modify the steady state value of the backlog. The difference between this new steady state value and the original value is called offset. The integral control is known to be effective in eliminating the offset. In our model, the proportional control eventually brings the state variable, net capacity discrepancy (Figure 5), to the same value, $D c_{t}=0$, as before the demand disturbance, after some fluctuations. Therefore, the P-control does not introduce an offset, and the integral control is not as useful as in other systems where a steady state offset occurs. However, in order to show benefits of integral control, we started with a PD control (with $K_{c}=1$, and $T_{d}=30$ ), and increase the I-control (by reducing the $T_{i}$ from very high number to $T_{i}$ of 300,100 and 30) as shown in Figure 11. As the $T_{i}$ value decreases to 100 from the very high value, the total costs actually improve from $\$ 1,592,000$ to $\$ 1,273,000$ as shown in Table 3. However, as $T_{i}$ decreases further to 30 , the oscillation of the backlog increases, and the total costs go to $\$ 2,376,000$. Therefore, as seen in previous sections for P-control and D-control, as I-control can improve overall control of system in certain situation, too much I-control will produce negative effects.

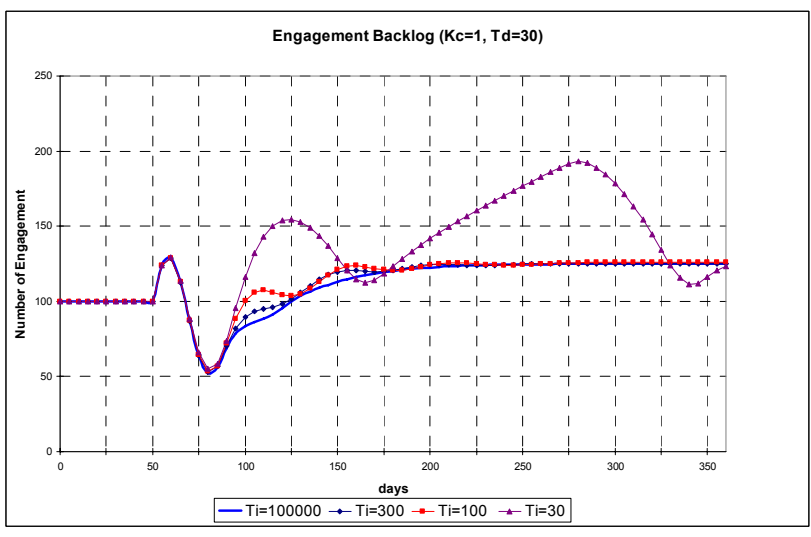

Figure 11. Effective of Integral Control

Table 3: Total Costs for Various I-Control Parameters

\begin{tabular}{|l|l|l|r|}
\hline P-Control & D-Control & I-Control & Costs \\
\hline$K_{c}=1$ & $T_{d}=30$ & None & $\$ 1,592,000$ \\
\hline$K_{c}=1$ & $T_{d}=30$ & $T_{i}=300$ & $\$ 1,407,000$ \\
\hline$K_{c}=1$ & $T_{d}=30$ & $T_{i}=100$ & $\$ 1,273,000$ \\
\hline$K_{c}=1$ & $T_{d}=30$ & $T_{i}=30$ & $\$ 2,376,000$ \\
\hline
\end{tabular}

\subsection{Effect of PID Control}

In this section, we attempted to find optimal PID control schemes by using an optimization capability available in Vensim, which uses Powell optimizer (Ventana Systems Inc 1998). The optimization result indicates that for the service business model, the optimal control is a PD control with $K_{c}=1.28$ and $T_{d}=9.88$ with no integral control as summarized in Table 4. The total costs for this optimal control scheme is $\$ 558,000$, which is the lower than any other combination of P, D, I control described earlier. Comparing with the simple proportional control $(\mathrm{Kc}=1)$ described in section 3.1, the total costs was reduced by $60 \%$ from $\$ 1,394,000$ to $\$ 558,000$.

Table 4: Total Costs for a PID Control

\begin{tabular}{|l|l|l|r|}
\hline P-Control & D-Control & I-Control & Costs \\
\hline$K_{c}=1.28$ & $T_{d}=9.88$ & None & $\$ 558,000$ \\
\hline
\end{tabular}

The profile of engagement backlog for the optimal control (Figure 12), having fewer oscillations and smaller amplitudes, looks much better than one with simple proportional control (Figure 4). Similar improvement is also observed for the net capacity discrepancy which also has fewer oscillations and smaller amplitudes as in Figure 13 than the one for simple proportional control (Figure 5). For the optimal control, the hiring and releasing is much better balanced (Figure 14) than the one for simple proportional control (Figure 6). 


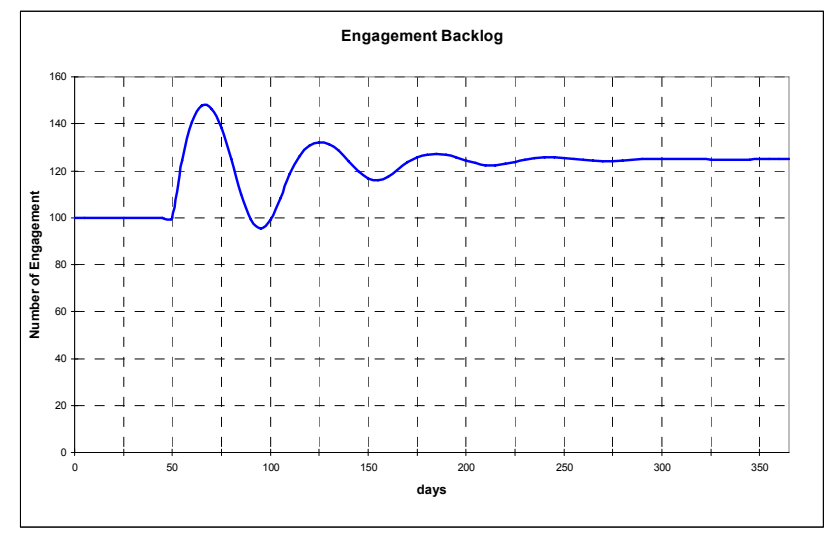

Figure 12: Engagement Backlog for a PID Control

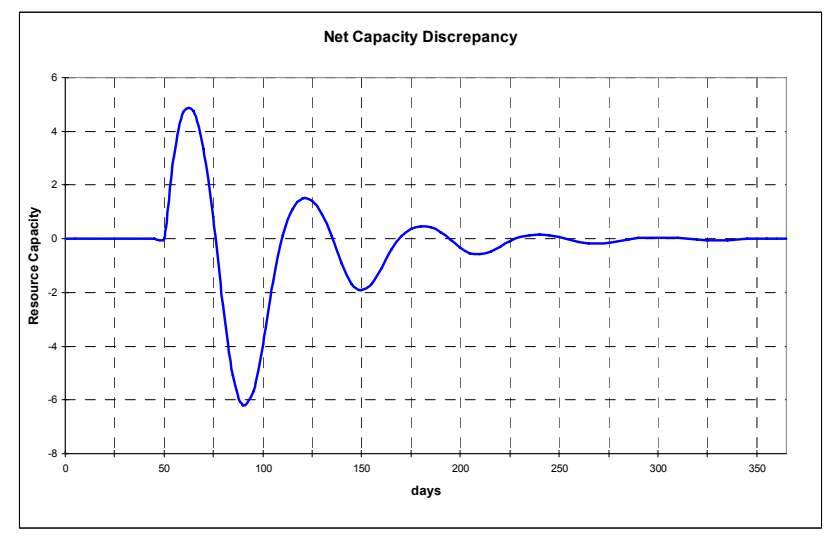

Figure 13: Net Capacity Discrepancy for a PID Control

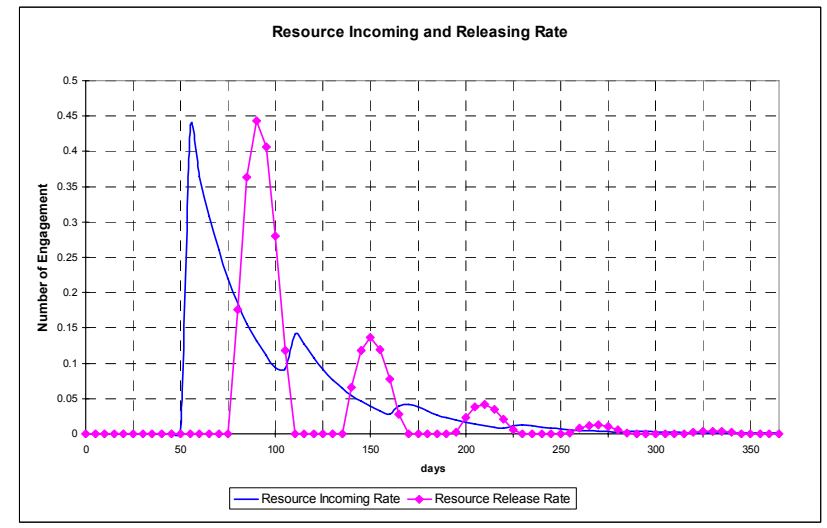

Figure 14: Resource Incoming Rate and Release Rate for a PID Control

\section{CLOSING REMARKS}

Demand disturbances in service business can be managed effectively by applying control theory to the resource actions such as hiring, releasing and cross training of workforce. Control theory has been used for many years in chemical and electrical engineering problem of controlling various processes, and recently in supply chain management problems. Managing workforce resource action for service business with a PID feedback control scheme is a good example of applying science to services, which have long been considered arts rather than sciences. Our study clearly indicates that effective PID feedback control can improve costs, utilization and stability of workforce in service business. The analysis and design of feedback control systems that incorporate P, I, D controllers is well-established; however, they have not been applied to the new area of service supply chains. We plan to extend our research by analyzing various demand disturbances in many service business environments, and generate useful insights that can lead to effective management of workforce.

\section{REFERENCES}

Anderson, E., Morrice, D., 1999. A Simulation Model To Study The Dynamics in a Service-Oriented Supply Chain, In Proceeding of the 1999 Winter Simulation Conference.

Anderson, E.G., and D.J. Morrice, 2000. "A Simulation Game for Service-Oriented Supply Chain Management: Does Information Sharing Help Managers with Service Capacity Decisions?" Production and Operations Management 9 (1): 44-55.

Akkermans, H, Vos, B. 2003. Amplification in Service Supply Chains: An Exploratory Case Study from the Telecome Industry, Production and Operations Management, 12(2): 204-223.

D’Azzo, J. J., and Houpis, C. H., 1981. Linear Control System Analysis and Design, Second Edition, McGraw-Hill, Second Edition.

Dejonckheere, J., Disney, S.M., Lambrecht, M.R. and Towill, D.R., 2004. "The impact of information enrichment on the bullwhip effect in supply chains: A control engineering perspective", European Journal of Operational Research, 153: 727-750.

Dietrich, B., Harrison, T., 2006. Servicing the Services, OR/MS Today, 33(3): 42-49.

Forrester, J. W. 1958. Industrial Dynamics: A Major Breakthrough for Decision Makers. Harvard Business Review, 36(4):37-66.

Lee, H., Padmanabhan, V., and Whang, S. 1997. Information Distortion in a Supply Chain: The Bullwhip Effect. Management Science, 43 (4):516-558.

Ortega, M., Lin., L. 2004. Control Theory Applications to the Production-Inventory Problem: a Review. International Journal of Production Research, 42(11), 2303-2322.

Simon, H.A., 1952. On the Application of Servomechanism Theory to The Study of Production Control, Econometrica, 20: 247-268. 
Sterman, J. D. 1989. Modeling Managerial Behavior: Misperceptions of Feedback in a Dynamic Decision Making Experiment. Management Science, 35 (3):321-339.

Sterman, J. D. 2000. Business dynamics: system thinking and modeling for a complex world. Boston: Irwin McGraw-Hill.

Ventana Systems, Inc. 1998. Vensim Version 3.0. 60 JacobGates Road, Harvard, MA 01451.

White, A.S., 1999, Management of Inventory Using Control Theory. International Journal of Technology management, 17: 847-860.

Wikner, J., Towill, D.R., and Naim, M.M. 1991. "Smoothing Supply Chain Dynamics", International Journal of Production Economics, 22: 231-248.

\section{AUTHOR BIOGRAPHIES}

YOUNG LEE is a Research Staff Member in the mathematical science department of IBM's T.J. Watson Research Center. Dr. Lee received B.S., M.S., and Ph.D. degrees in chemical engineering from Columbia University. He joined the IBM Research Division in 2002, and has been working in the areas of supply chain simulation and optimization. Prior to joining IBM, he had worked for BASF for 14 years, where he had founded and managed the Mathematical Modeling Group, and led development of numerous optimization and simulation models for various logistics and manufacturing processes. His research interest includes simulation and optimization of supply chain, manufacturing, services and business processes. His email address is <ymleedus. i bm . com>.

LIANJUN AN is a Researcher in the mathematical science department of IBM's T.J. Watson Research Center. He received B.S., and M.S. degrees in mathematics from Tsinghua University of China, and Ph.D. degree in applied mathematics from Duke University. He worked in McMaster and Stony Brook Universities and conducted nonlinear analysis and parallel computation on partial differential equation models describing elastoplastic deformation and transport phenomena in porous media. $\mathrm{He}$ then subsequently joined IBM since 1998 and has been working in the areas of system dynamics modeling and simulation, operational forecasting and optimization, business process modeling and decision support system. His email address is <alianjun@us.ibm.com>.

DANIEL CONNORS is a Research Staff Member in the Business Analytics and Mathematical Science Department at the IBM T. J. Watson Research Center. He received the B.S.E. degree in Electrical Engineering from the University of Michigan in 1982, the M.S. and Ph. D. degrees in Electrical Engineering from the University of Illinois in 1984 and 1988, respectively. He joined the
IBM Research Division in 1988. His research interests include modeling, simulation and design of business processes and design of decision support tools for manufacturing, supply chain logistics and workforce management. 